\title{
Two (Multi) Point Nonlinear Lyapunov Systems Associated with an $n$th Order Nonlinear System of Differential Equations - Existence and Uniqueness
}

\author{
K.N. MURTY ${ }^{a}{ }^{*}$, G.W. HOWELL ${ }^{a}$ and G.V.R.L. SARMA ${ }^{b}$ \\ a Mathematical Sciences, Florida Institute of Technology, Melbourne, \\ FL 32901-6975, USA; ' ${ }^{\circ}$ Department of Applied Mathematics, Andhra University, \\ Visakhapatnam - 530003, India
}

(Received 15 February 2000)

This paper presents a criterion for the existence and uniqueness of solutions to two and multipoint boundary value problems associated with an $n$th order nonlinear Lyapunov system. A variation of parameters formula is developed and used as a tool to obtain existence and uniqueness. We discuss solution of the second order problem by the ADI method and develop a fixed point method to find the general solution of the $n$th order Lyapunov system. The results of Barnett (SIAM J. Appl. Anal. 24(1), 1973) are a particular case.

Keywords: Lyapunov systems; Boundary value problems; Two point and multipoint nonlinear systems; Kronecker products; Fundamental matrix solutions

Classification Categories: 34D10

\footnotetext{
*Corresponding author. Tel.: 321-674-8091. E-mail: murty@fit.edu.
} 


\section{INTRODUCTION}

This paper gives a criterion for the existence and uniqueness of the solution to a nonlinear $n$th order system of differential equations

$$
\begin{aligned}
T^{(n)}(t)= & A^{n} T(t)+\left(\begin{array}{c}
n \\
1
\end{array}\right) A^{n-1} T(t) B+\left(\begin{array}{c}
n \\
2
\end{array}\right) A^{n-2} T(t) B^{2}+\cdots \\
& +\left(\begin{array}{l}
n \\
r
\end{array}\right) A^{n-r} T(t) B^{r}+\cdots+T(t) B^{n}+F(t, T(t)) \\
= & \sum_{r=0}^{n}\left(\begin{array}{l}
n \\
r
\end{array}\right) A^{n-r} T(t) B^{r}+F(t, T(t)), \quad a \leq t \leq b
\end{aligned}
$$

with $A^{0}=B^{0}=I$ and where $A, B$ and $T(t)$ are square matrices of order $n$. We assume that $A$ and $B$ are constant matrices, that $T$ is a variable matrix whose components are continuous functions on $[a, b]$, and that the nonlinear function $F:[a, b] \times \mathcal{R}^{n \times n} \rightarrow \mathcal{R}^{n \times n}$ is continuous. We further assume that $F(t, 0) \equiv 0$ on $[a, b]$. This restriction only ensures (1.1) admits a trivial solution. We in fact seek a solution of (1.1) satisfying the boundary condition matrix

$$
M T(a)+N T(b)=\alpha
$$

(or $\sum_{i=1}^{n} M_{i} T\left(t_{i}\right)=\alpha$ ). Here $a=t_{1}<t_{2}<\cdots<t_{n}=b$ where $M$ and $N$ are constant square matrices of order $n$ and all scalars are assumed to be real. For $n=1$, the problem reduces to

$$
T^{\prime}(t)=A T(t)+T(t) B
$$

The boundary value problem (1.1), (1.2) naturally arises in a number of areas of applied mathematics and control engineering. It has attracted the attention of such mathematicians as Atkinson [1], Bellman [3], and others with the closed form solution given by Murty et al. in [6].

This paper is organized as follows: in Section 2, we present the general solution of (1.1), (1.2) and develop variation of parameters formula for the nonhomogenous system (1.1). With this formula as a tool, we develop in Section 3 our main results on existence and uniqueness of solutions to two- and multi-point boundary value problems. The results presented in this paper pave the way for studying problems of stability for nonlinear systems of higher order matrix systems. 
In Section 4, we deduce the results of Barnett given in [2] as a particular case of our results and draw certain natural interesting conclusions.

\section{GENERAL SOLUTION OF THE MATRIX SYSTEM}

In this section, we establish the general solution of nonlinear matrix Lyapunov system in terms of the fundamental matrix solutions. The results presented here include those of Barnett [2] as a special case. We assume throughout that $Y$ is a fundamental matrix solution of $T^{\prime}=$ $A T$ and $Z$ is a fundamental matrix solution of $T^{\prime}=B^{*} T$. ( ${ }^{*}$ refers to the transpose of the complex conjugate matrix of $B$ ). We present the following theorems.

THEOREM 2.1 If $Y$ is a fundamental matrix solution of $T^{\prime}=A T$, then $Y$ is also a fundamental matrix solution of $T^{(n)}(t)=A^{n} T(t)$, where $A$ is an $n \times n$ matrix.

Proof $Y$ is a fundamental matrix solution of $T^{\prime}=A T$ if and only if $Y^{\prime}(t)=A Y(t)$. This implies $Y^{\prime \prime}(t)=A Y^{\prime}(t)=A^{2} Y(t)$. Again $Y^{\prime \prime \prime}(t)=$ $A^{2} Y^{\prime}(t)=A^{3} Y(t)$ and so on. Thus the proof is complete.

THEOREM 2.2 If $Y(t)$ is a fundamental matrix solution of $T^{\prime}=A T$, then for any real or complex constant $n, Y(n t)$ is a fundamental matrix solution of $T^{\prime}=(n A) T$.

Proof The proof is a direct verification and hence omitted.

THEOREM 2.3 Any solution of

$$
T^{(n)}(t)=\sum_{r=0}^{n}\left(\begin{array}{l}
n \\
r
\end{array}\right) A^{n-r} T(t) B^{r}
$$

is of the form $T(t)=Y(t) \mathcal{C} Z^{*}(t)$, where $\mathcal{C}$ is a constant square matrix of order $n$.

Proof We first note that by the Leibnitz theorem on the derivative of the product of two functions

$$
\begin{aligned}
\left(Y(t) C Z^{*}(t)\right)^{(n)}= & Y^{(n)}(t) C Z^{*}(t)+\left(\begin{array}{c}
n \\
1
\end{array}\right) Y^{n-1}(t) C Z^{*^{\prime}}(t)+\cdots \\
& +\left(\begin{array}{l}
n \\
r
\end{array}\right) Y^{n-r}(t) C Z^{*^{(r)}}(t)+\cdots+Y(t) C Z^{*^{(n)}}(t) .
\end{aligned}
$$


Using Theorem 2.1, we have $Y^{(n)}(t)=A^{n} Y(t), Y^{(n-1)}(t)=A^{n-1} Y(t)$, etc. we can easily verify that $T(t)$ defined by $T(t)=Y(t) \mathcal{C} Z^{*}(t)$ is a solution of (2.1).

To prove that every solution of (2.1) is of this form, let $T$ be a solution and let $K$ be a square matrix of order $n$ defined by $K(t)=$ $Y^{-1}(t) T(t)$. Now $T(t)=Y(t) K(t)$. Hence

$$
\begin{aligned}
T^{(n)}(t)= & Y^{(n)}(t) K(t)+\left(\begin{array}{c}
n \\
1
\end{array}\right) Y^{(n-1)}(t) K(t)+\cdots \\
& +\left(\begin{array}{l}
n \\
r
\end{array}\right) Y^{n-r}(t) K+Y(t) K^{(n)}(t) \\
= & A^{n} Y(t) K(t)+A^{n-1} Y(t) K(t)+\cdots \\
& +\left(\begin{array}{l}
n \\
r
\end{array}\right) A^{n-r} Y(t) K^{(r)}(t)+\cdots+Y(n) K^{(n)}(t) .
\end{aligned}
$$

This is true if and only if $K^{*}(t)=Z \mathcal{C}, K^{*^{\prime}}(t)=Z^{*^{\prime}} \mathcal{C}, \ldots, K^{*^{n}}(t)=Z^{*^{n}} \mathcal{C}$ or $K(t)=\mathcal{C}^{*} Z^{*}$. Hence $T(t)=Y(t) K(t)=Y(t) \mathcal{C} Z^{*}(t)\left(\right.$ Take $\left.\mathcal{C}^{*}=\mathcal{C}\right)$.

THEOREM 2.4 Any solution of the nonlinear matrix Lyapunov system

$$
\begin{aligned}
T^{(n)}(t)= & A^{n} T+\left(\begin{array}{c}
n \\
1
\end{array}\right) A^{n-1} T B+\cdots \\
& +\left(\begin{array}{l}
n \\
r
\end{array}\right) A^{n-r} T B^{r}+\cdots+T B^{n}+F(t, T(t))
\end{aligned}
$$

is of the form $T(t)=Y(t) \mathcal{C} Z^{*}+\bar{T}(t)$, where $\bar{T}(t)$ is a particular solution of (2.2).

Proof It can be easily verified that $T$ defined by $T(t)=Y(t) \mathcal{C} Z^{*}(t)+$ $\bar{T}(t)$ is a solution of (2.2). To prove that every solution is of this form, let $T$ be any solution of (2.2) and $\bar{T}(t)$ be a particular solution of (2.2). The $T-\bar{T}$ is a solution of the homogeneous equation

$$
T^{(n)}=\sum_{r=0}^{n}\left(\begin{array}{l}
n \\
r
\end{array}\right) A^{n-r} T B^{r}
$$

with $A^{0}=B^{0}=I$. Hence by Theorem $2.1, T-\bar{T}=Y C Z^{*}$ or $T=$ $\bar{T}+Y C Z^{*}$, and the proof is complete.

The next theorem establishes the variation of parameters formula for the $n$th order nonlinear nonhomogeneous matrix differential system. 
THEOREM 2.5 Let $Y$ be a fundamental matrix solution of $T^{\prime}=A T$ and $Z$ be a fundamental matrix solution of $T^{\prime}=B^{*} T$. Further suppose that the variable matrix $\mathcal{C}$ is such that

$$
\left(Y^{\prime} \mathcal{C}^{\prime} Z^{*}+Y \mathcal{C}^{\prime} Z^{*}\right)^{i-2}=0 \text { for each } i=2,3, \ldots, n
$$

Then a particular solution of (2.2) is of the form

$$
\begin{aligned}
\bar{T}(t)=Y(t)[ & \int_{a}^{t} \int_{a}^{\tau_{1}} \int_{a}^{\tau_{2}} \cdots \int_{a}^{\tau_{n-1}} Y^{-1}(s) F(s, T(s)) . \\
& \left.\times Z^{*-1}(s) \mathrm{d} s, \mathrm{~d} \tau_{n-1} \mathrm{~d} \tau_{n-2} \cdots \mathrm{d} \tau_{1}\right] Z^{*}(t) .
\end{aligned}
$$

Proof Any solution of the homogeneous matrix Lyapunov system is of the form $T(t)=Y(t) \mathcal{C} Z^{*}(t)$, where $\mathcal{C}$ is an $n \times n$ constraint matrix. Since such a solution cannot be a solution of the nonlinear nonhomogeneous system (2.2) unless $F(t, T(t))=0$. We assume that $\mathcal{C}$ is a variable matrix and seek a particular solution of the form $\bar{T}(t)=$ $Y(t) \mathcal{C}(t) Z^{*}(t)$. Substituting the general form of $\bar{T}(t)$ in (2.2) and on using (2.3), we get

$$
Y(t) \mathcal{C}^{(n)}(t) Z^{*}(t)=F(t, T(t))
$$

or

$$
\mathcal{C}^{(n)}(t)=Y^{-1}(t) F(t, T(t)) Z^{*(-1)}(t) .
$$

Upon integrating $n$ times, we get

$$
\begin{aligned}
\mathcal{C}= & \int_{a}^{t} \int_{a}^{\tau_{1}} \int_{a}^{\tau_{2}} \cdots \int_{a}^{\tau_{n-1}} Y^{-1}(s) F(s, T(s)) \\
& \times Z^{*(-1)}(s) \mathrm{d} s \mathrm{~d} \tau_{n-1} \mathrm{~d} \tau_{n-2} \cdots \mathrm{d} \tau_{1} .
\end{aligned}
$$

It can be easily verified that

$$
\begin{array}{r}
\bar{T}(t)=Y(t)\left[\int_{a}^{t} \int_{a}^{\tau_{1}} \int_{a}^{\tau_{2}} \cdots \int_{a}^{\tau_{n-1}} Y^{-1}(s) F(s, T(s))\right. \\
\left.\times Z^{*-1}(s) \mathrm{d} s \mathrm{~d} \tau_{n-1} \mathrm{~d} \tau_{n-2} \cdots \mathrm{d} \tau_{1}\right] Z^{*}(t)
\end{array}
$$

is a solution of (2.2), and hence the proof of the theorem is complete. 
THEOREM 2.6 Let $T$ be any solution of (2.2) and $\bar{T}$ be a particular solution of (2.2). Then any solution of (2.2) is of the form

$$
\begin{aligned}
T(t)=Y(t) \mathcal{C} Z^{*}(t)+Y(t)[ & \int_{a}^{t} \int_{a}^{\tau_{1}} \int_{a}^{\tau_{2}} \cdots \int_{a}^{\tau_{n-1}} Y^{-1}(s) F(s, T(s)) \\
& \left.\times Z^{*(-1)}(s) \mathrm{d} s \mathrm{~d} \tau_{n-1} \mathrm{~d} \tau_{n-2} \cdots \mathrm{d} \tau_{1}\right] Z^{*}(t) .
\end{aligned}
$$

Proof It can be easily verified that $T$ defined by (2.5) is a solution of (2.2). Further, if $T$ is any solution of (2.2) and $\bar{T}(t)$ is a particular solution of (2.2), then $T-\bar{T}$ is a solution of $T^{(n)}(t)=\sum_{r=0}^{n}\left(\begin{array}{l}n \\ r\end{array}\right) A^{n-r} T B^{r}$. Any solution of the homogeneous systems is of the form $Y(t) C Z^{*}(t)$, where $\mathcal{C}$ is an $n \times n$ constant matrix. Thus, or $T-\bar{T}=Y C Z^{*}$ or $T=$ $\bar{T}+Y C Z^{*}$. By Theorem 2.3, any solution $T$ is of the form $T(t)=$ $\bar{T}(t)+Y(t) \mathcal{C} Z^{*}(t)$, where $\bar{T}(t)$ is given by (2.4). Thus the proof of the theorem is complete.

Next, we consider the boundary value problem

$$
T^{(n)}(t)=\sum_{r=0}^{n}\left(\begin{array}{l}
n \\
r
\end{array}\right) A^{n-r} T B^{r}+F(t, T(t))
$$

satisfying the general matrix boundary conditions

$$
M T(a)+N T(b)=\alpha
$$

or

$$
\sum_{i=1}^{n} M_{i} T\left(t_{i}\right)=\alpha
$$

Recall that any solution $T$ of the nonhomogenous matrix Lyapunov system (2.2) is of the form

$$
\begin{aligned}
T(t)=Y(t) \mathcal{C} Z^{*}(t)+Y(t)[ & \int_{a}^{t} \int_{a}^{\tau_{1}} \int_{a}^{\tau_{2}} \cdots \int_{a}^{\tau_{n-1}} Y^{-1}(s) F(s, T(s)) \\
& \left.\times Z^{*(-1)}(s) \mathrm{d} s \mathrm{~d} \tau_{n-1} \mathrm{~d} \tau_{n-2} \cdots \mathrm{d} \tau_{1}\right] Z^{*}(t) .
\end{aligned}
$$


Substituting the general form of $T(t)$ in the boundary condition matrix (2.7), we get

$$
\begin{aligned}
& M Y(a) \mathcal{C} Z^{*}(a)+N Y(b) \mathcal{C} Z^{*}(b)+N Y(b)\left[\int_{a}^{b} \int_{a}^{\tau_{1}} \int_{a}^{\tau_{2}} \cdots \int_{a}^{\tau_{n-1}} Y^{-1}(s)\right. \\
& \left.\quad \times F(s, T(s)) Z^{*(-1)}(s) \mathrm{d} s \mathrm{~d} \tau_{n-1} \mathrm{~d} \tau_{n-2} \cdots \mathrm{d} \tau_{1}\right] Z^{*}(b)=\alpha
\end{aligned}
$$

or

$$
M Y(a) C Z^{*}(a)+N Y(b) \mathcal{C} Z^{*}(b)=\mathcal{K},
$$

where

$$
\begin{aligned}
\mathcal{K}=\alpha-N Y(b)[ & \int_{a}^{b} \int_{a}^{\tau_{!}} \int_{a}^{\tau_{2}} \cdots \int_{a}^{\tau_{n-1}} Y^{-1}(s) F(s, T(s)) \\
& \left.\times Z^{*-1}(s) \mathrm{d} s \mathrm{~d} \tau_{n-1} \mathrm{~d} \tau_{n-2} \cdots \mathrm{d} \tau_{1}\right] Z^{*}(b)
\end{aligned}
$$

which is equivalent to

$$
A_{1} \mathcal{C} B_{1}+A_{2} \mathcal{C} B_{2}=\mathcal{K},
$$

where $A_{1}=M Y(a), A_{2}=N Y(b), B_{1}=Z^{*}(a)$ and $B_{2}=Z^{*}(b)$. We find the general solution $\mathcal{C}$ of (2.11) in terms of the known matrices $A_{1}, A_{2}$, $B_{1}, B_{2}$, and $\mathcal{K}$. In the case of the multi-point boundary value problem (2.2) satisfying (2.8), we get

$$
\begin{aligned}
\sum_{i=1}^{n} M_{i} Y\left(t_{i}\right) \mathcal{C} Z^{*}\left(t_{i}\right)= & \alpha-\sum_{i=2}^{n} M_{i} Y\left(t_{i}\right)\left[\int_{a}^{t} \int_{a}^{\tau_{1}} \int_{a}^{\tau_{2}} \cdots \int_{a}^{\tau_{n-1}} Y^{-1}(s)\right. \\
& \left.\times F(s, T(s)) Z^{*-1}(s) \mathrm{d} s \mathrm{~d} \tau_{n-1} \mathrm{~d} \tau_{n-2} \cdots \mathrm{d} \tau_{1}\right] Z^{*}\left(t_{i}\right)
\end{aligned}
$$

which is equivalent to

$$
\sum_{i=1}^{n} A_{i} \mathcal{C} B_{i}=\mathcal{K}
$$

where $A_{i}=M_{i} Y\left(t_{i}\right)$ and $B_{i}=Z^{*}\left(t_{i}\right)$. 


\section{ANALYSIS OF THE MATRIX $\mathcal{C}$ AND THE GENERAL SOLUTION $T(t)$}

In this section, we shall be concerned with the general form of the constant matrix $\mathcal{C}$ satisfying Eq. (2.11):

$$
A_{1} \mathcal{C} B_{1}+A_{2} C B_{2}=\mathcal{K}
$$

or

$$
\sum_{i=0}^{n-1} A^{i} \mathcal{C} B_{i}=\mathcal{K}
$$

Transformation to a vector equation, using the Kronecker product of matrices, allows us to use currently available numerical techniques for the solution of (2.11) or (3.1). We use the following notation. If $A \in \mathcal{R}^{n \times n}$ and $B \in \mathcal{R}^{n \times n}$, then their Kronecker product $A \otimes B \in \mathcal{R}^{n^{2} \times n^{2}}$ is defined as

$$
A \otimes B=a_{i j} B \quad \text { for } i, j=1,2, \ldots, n .
$$

We now consider our target Eqs. (2.11) or (3.1). A system $A \mathcal{C}=X$ is solvable for $\mathcal{C}$ if the eigenvalues $\lambda_{i}$ of $A$ are all nonzero. Whether the system (3.1) is uniquely solvable depends on the eigenvalues of $A$ and on a polynomial matrix

$$
B(x)=\sum_{i=1}^{n} B_{i} x^{i}, \quad x \in \mathcal{C} .
$$

THEOREM 3.1 Equation (3.1) has a unique solution $\mathcal{C}$ if and only if

$$
\operatorname{det} B\left(\lambda_{i}\right) \neq 0 \text { for } i=1,2, \ldots, n \text {. }
$$

Proof The left-hand side of (3.1) will be regarded as the product of $\mathcal{C}$ and $B$. For that reason, we define $F=\sum_{i} F_{i} x^{i} \in \mathcal{C}^{n \times n}$ and for $M \in \mathcal{R}^{n \times n}$, the operation $M * F=\sum_{i} A^{i} M F_{i}$. Then $\mathcal{C}^{n \times n}$ becomes a right module over the ring $\mathcal{C}^{n \times n}[x]$. Let $a(x)$ be the characteristic polynomial of $A$, and take

$$
\Phi=a(x) \mathcal{C}^{n \times n}[x]
$$

so that $\Phi$ is the ideal in $\mathcal{C}^{n \times n}[x]$ generated by $a(x) I$. By the CayleyHamilton theorem, we have $M * H=0$ for all $H \in \Phi$. Hence we can 
define

$$
M *(F+\Phi)=M * F .
$$

With this fact, we can rewrite (3.1) as

$$
\mathcal{C} *(B+\Phi)=\mathcal{K} .
$$

It is clear that (3.2) is solvable if and only if $B+\Phi$ is invertible in the quotient ring $\mathcal{C}^{n \times n}[x] / \Phi[5]$.

THEOREM 3.2 If the system of Eqs. (3.1) is universally solvable and

$$
G(x)=\sum_{i=1}^{n} G_{i} x^{i}
$$

is the polynomial given by

$$
B G=G B=I(\bmod \Phi), \operatorname{deg} G<n
$$

then

$$
\mathcal{C}=\sum_{i=1}^{n} A^{i} \mathcal{K} G_{i}
$$

is the unique solution of (3.1).

Proof The coefficient matrices $G_{i}$ of (3.3) can be determined from an auxiliary equation which involves the companion matrix $F_{\alpha}$ of $a(x)$. Substituting the general form of $\mathcal{C}$, we get

$$
\begin{aligned}
T(t)= & Y(t)\left[\sum_{i=1}^{n} A^{i} \mathcal{K} G_{i} Z^{*}(t)\right]+Y(t)\left[\int_{a}^{t} \int_{a}^{\tau_{1}} \int_{a}^{\tau_{2}} \cdots \int_{a}^{\tau_{n-1}} Y^{-1}(s)\right. \\
& \left.\times F(s, T(s)) Z^{*(-1)}(s) \mathrm{d} s \mathrm{~d} \tau_{n-1} \mathrm{~d} \tau_{n-2} \cdots \mathrm{d} \tau_{1}\right] Z^{*}(t)
\end{aligned}
$$

is the unique solution of the multipoint boundary value problem (1.1), (1.2), $i=1,2, \ldots, n$. 
When the matrices $A_{i}$ are pairwise commutative, the general case (2.12) can be approached with a somewhat more complicated theoretical treatment [5].

We now turn our attention to the boundary value problem (1.1), (1.2). For this problem, we need to solve for $\mathcal{C}$ from Eq. (2.11)

$$
A_{1} \mathcal{C} B_{1}+A_{2} C B_{2}=\mathcal{K},
$$

a problem for which practical numeric techniques have been developed. If $A_{1}$ and $B_{2}$ are invertible, then

$$
A \mathcal{C}+\mathcal{C} B=\mathcal{K},
$$

where $A=A_{1}^{-1} A_{2}, B=B_{1} B_{2}^{-1}$, and $\mathcal{K}=A_{1}^{-1} \mathcal{K} B_{2}^{-1}$. Equation (3.4) is known as the Sylvester matrix equation. Let $\left\{\lambda_{i}\right\}$ be the set of eigenvalues of $A$ and $\left\{\mu_{i}\right\}$ the set of eigenvalues of $B$. If there is no choice of $i, j, 1 \leq i, j \leq n$ such that $\lambda_{i}+\mu_{j}=0$ then (3.4) has a unique solution $\mathcal{C}$ for any choice of $\mathcal{K}$. The system is then said to be nonsingular. Reduction of one of $A$ and $B$ to tridiagonal form and the rest to digaonal form via the symmetric $Q R$ algorithm provides a robust and elegant algorithm for solution of the Sylvester equation when both $A$ and $B$ are symmetric. On the other hand, when $A$ or $B$ are not symmetric, the Householder reduction to Hessenberg form does not yield a tridiagonal matrix. The method of Golub et al. [4] requires for the reduction of only one of the matrices $A$ or $B$ to Schur (upper triangular) form. Considerable savings in computations may be made through the use of Gaussian reduction to banded form and then using Alternating Direction Implicit (ADI) solution of the reduced equation.

Use similarity transformations $G$ and $H$, respectively, to reduce $A$ and $B$ to the banded matrices $S$ and $T$. Then the Sylvester equation (3.4) reduces to

$$
S Z+Z T=F,
$$

where

$$
\begin{aligned}
& S=G A G^{-1}, \\
& T=H B H^{-1}, \\
& F=G \mathcal{K} G^{-1},
\end{aligned}
$$


and

$$
Z=G C H^{-1}
$$

respectively. Once $A$ and $B$ have been reduced to $S$ and $T$ of small bandwidth, one can solve the Eq. (3.4) by ADI iteration [7]. The iteration equation for the reduced system is as follows

$$
\begin{gathered}
Z_{0}=0 \\
\left(S+p_{j} I_{n}\right) Z_{j-1 / 2}=F-Z_{j-1}\left(T-p_{j} I_{m}\right), \\
\left(T^{*}+q_{j} I_{n}\right) Z_{j}^{*}=\left[F-\left(S-q_{j} I_{n}\right) Z_{j-1 / 2}\right]^{*} .
\end{gathered}
$$

Let the right-hand side of Eqs. (3.11) and (3.12) be denoted by $G_{j-1 / 2}$ and $G_{j}$. The ADI iteration first computes

$$
G_{1 / 2}=F
$$

and thereafter on the half-steps

$$
G_{j-1 / 2}=F+\left[p_{j} I i_{m}+q_{j-1} Z_{j-1}-G_{j-1}\right]^{\mathrm{T}} .
$$

For the whole steps

$$
G_{j-1}=F+\left[p_{j} I_{m}+q_{j} Z_{j-1 / 2}-G_{j-1 / 2}\right]^{\mathrm{T}}
$$

where $\mathrm{T}$ refers to the transpose of the matrix.

A method of computing the solution of (1.1) satisfying (1.2) is given in [6] for first order systems of differential equations. We now present the same technique for the $n$th order nonlinear Lyapunov system (1.1). We assume that $F$ satisfies a Lipschitz condition on $[a, b] \times \mathcal{R}^{n \times n}$, i.e.,

$$
\left\|F\left(s, T i_{1}(s)\right)-F\left(s, T_{2}(s)\right)\right\| \leq L\left\|T_{1}-T_{2}\right\|(L>0)
$$

and define

$$
\operatorname{cond}(Y)=\max _{t \in[a, b]}\|Y(t)\| \max _{t \in[a, b]}\left\|Y^{-1}(t)\right\|
$$

and

$$
\operatorname{cond}(Z)=\max _{t \in[a, b]}\|Z(t)\| \max _{t \in[a, b]}\left\|Z^{-1}(t)\right\| .
$$


If $A_{1}$ and $B_{1}$ are nonsingular, then (2.11) is equivalent to

$$
\mathcal{C}-A C B=Y,
$$

where $A=-\left(A_{1}^{-1} A_{2}\right), B=\left(B_{2} B_{1}^{-1} A^{-1}\right)$ and $Y=A_{1}^{-1} \mathcal{K} B_{1}^{-1}$. Now, to solve for $\mathcal{C}$ we have the following analysis:

$$
\begin{aligned}
\mathcal{C}-A C B=Y & \Leftrightarrow\left[I \otimes I-\left(A \otimes B^{\mathrm{T}}\right)\right] \mathcal{C}=\mathcal{Y} \\
& \Leftrightarrow I c-\left(A \otimes B^{\mathrm{T}}\right) c=y \\
& \Leftrightarrow I c-G c=y, \quad \text { where } G=\left(A \otimes B^{\mathrm{T}}\right) .
\end{aligned}
$$

Putting $\mathcal{C}=Y+A C B$ in the second term of (3.14), we have the following equivalent statements:

$$
\begin{aligned}
\mathcal{C}-A(Y+A \mathcal{C} B) B=Y & \Leftrightarrow c-G(y+G c)=y, \\
\mathcal{C}-A^{2} \mathcal{C} B^{2}=Y+A Y B & \Leftrightarrow c-G^{2} c=y+G y, \\
\mathcal{C}-A^{3} \mathcal{C} B^{3}=Y+A Y B+A^{2} Y B^{2} & \Leftrightarrow c-G^{3} c=y+G y+G^{2} y,
\end{aligned}
$$

$\mathcal{C}-A^{n} \mathcal{C} B^{n}$

$$
\begin{aligned}
=Y+A Y B+\cdots+A^{n-1} Y B^{n-1} \Leftrightarrow c-G^{n} c= & y+G y+G^{2} y \\
& +\cdots+G^{n-1} y .
\end{aligned}
$$

If the spectral radii of $A$ and $B$ satisfy

$$
\rho(A) \rho(B)<1,
$$

then $A^{n} Y B^{n} \rightarrow 0$ as $n \rightarrow \infty$. In this case

$$
\begin{aligned}
\mathcal{C} & =Y+\sum_{j=1}^{\infty} A^{j} Y B^{j} \\
& =A_{1}^{-1} \mathcal{K} B_{1}^{-1}+\sum_{j=1}^{\infty}\left(A_{1}^{-1} A_{2}\right)^{j}\left(A_{1} \mathcal{K} B_{1}\right)\left(B_{2} B_{1}^{-1}\right)^{j} .
\end{aligned}
$$


Substituting the general form of $\mathcal{C}$ in (2.11), we get

$$
\begin{aligned}
T(t)= & Y(t) A_{1}^{-1} \mathcal{K} B_{1}^{-1}+\sum_{j=1}^{\infty}\left(A_{1}^{-1} A_{2}\right)^{j}\left(A_{1} \mathcal{K} B_{1}\right) \\
& \times\left(B_{2} B_{1}^{-1}\right)^{j} Z^{*}(t)+Y(t)\left[\int_{a}^{t} \int_{a}^{\tau_{1}} \int_{a}^{\tau_{2}} \cdots \int_{a}^{\tau_{n-1}} Y^{-1}(s)\right. \\
& \left.\times F(s, T(s)) Z^{*-1} \mathrm{~d} s \mathrm{~d} \tau_{n-1} \mathrm{~d} \tau_{n-2} \cdots \mathrm{d} \tau_{1}\right] Z^{*}(t) \\
= & Y(t) A_{1}^{-1}\left[\alpha-N Y(b)\left[\int_{a}^{b} \int_{a}^{\tau_{1}} \int_{a}^{\tau_{2}} \cdots \int_{a}^{\tau_{n-1}} Y^{-1}(s)\right.\right. \\
& \left.\left.\times F(s, T(s)) \mathrm{d} s \mathrm{~d} \tau_{n-1} \mathrm{~d} \tau_{n-2} \cdots \mathrm{d} \tau_{1}\right]\right] B_{1}^{-1} Z^{*}(b) \\
& -Y(t) B_{1}^{-1}+\sum_{j=1}^{\infty}\left(A_{1}^{-1} A_{2}\right)^{j}\left(A_{1}[\alpha-N Y(b)\right. \\
& \times\left[\int_{a}^{b} \int_{a}^{\tau_{1}} \int_{a}^{\tau_{2}} \cdots \int_{a}^{\tau_{n-1}} Y^{-1}(s)\right. \\
& \left.\left.\left.\times F(s, T(s)) \mathrm{d} s \mathrm{~d} \tau_{n-1} \mathrm{~d} \tau_{n-2} \cdots \mathrm{d} \tau_{1}\right]\right] B_{1}\right)\left(B_{2} B_{1}^{-1}\right)^{j} Z^{*}(t) \\
& +Y(t)\left[\int_{a}^{t} \int_{a}^{\tau_{1}} \int_{a}^{\tau_{2}} \cdots \int_{a}^{\tau_{n-1}} Y^{-1}(s)\right. \\
& \left.\times F(s, T(s)) Z^{*-1} \mathrm{~d} s \mathrm{~d} \tau_{n-1} \mathrm{~d} \tau_{n-2} \cdots \mathrm{d} \tau_{1}\right] Z^{*}(t) .
\end{aligned}
$$

To obtain a unique solution to the two point boundary value problem, we define the operator $T$ by

$$
\begin{aligned}
T^{i}(t)= & Y(t) B Z^{*}(t)-Y(t) A_{1}^{-1} N Y(b) \int_{a}^{b} \int_{a}^{\tau_{1}} \int_{a}^{\tau_{2}} \cdots \int_{a}^{\tau_{n-1}} Y^{-1}(s) \\
& \times F(s, T(s)) Z^{*^{-1}}(s) \mathrm{d} s \mathrm{~d} \tau_{n-1} \mathrm{~d} \tau_{n-2} \cdots \mathrm{d} \tau_{1} \\
& \times B_{1}^{-1}\left(B_{s} B_{1}^{-1}\right)^{j} Z^{*}(t)+\int_{a}^{b} \int_{a}^{\tau_{1}} \int_{a}^{\tau_{2}} \cdots \int_{a}^{\tau_{n-1}} Y^{-1}(s) \\
& \times F\left(s, T^{(i-1)}(s) Z^{*-1}(s)\right) \mathrm{d} s \mathrm{~d} \tau_{n-1} \cdots \mathrm{d} \tau_{1} Z^{*}(t) .
\end{aligned}
$$


Consider

$$
\begin{aligned}
\left\|T^{i}(t)-T^{i-1}(t)\right\| \leq & \operatorname{Cond}(Y) \operatorname{Cond}(Z)\left\{\sum_{j=1}^{\infty}\left\|A_{1}^{-1} A_{2}\right\|^{j} .\right. \\
& \left.\times\left\|A_{1}^{-1}\right\|\|N Y(b)\|\left\|B_{1}^{-1}\right\|\left\|B_{2} B_{1}^{-1}\right\|^{j}+1\right\} \frac{L(b-a)^{n}}{n !} .
\end{aligned}
$$

Let

$$
\alpha=\frac{\operatorname{Cond}(Y) \operatorname{Cond}(Z)}{1-\left\|A_{1}^{-1} A_{2}\right\|\left\|B_{2} B_{1}^{-1}\right\|}<L\left[\left\|A_{1}^{-1}\right\|\left\|B_{1}^{-1}\right\|\|N Y(b)\|+1\right]<1 .
$$

Then

$$
\begin{aligned}
\left\|T^{i}(t)-T^{i-1}(t)\right\| & <\alpha\left\|T^{i-1}(t)-T^{i-2}(t)\right\| \frac{(b-a)^{i}}{i !} \\
& \leq \alpha^{2}\left\|T^{i-2}(t)-T^{i-3}(t)\right\| \frac{(b-a)^{i+1}}{(i+1) !} \\
& \vdots \\
& \leq \alpha^{n}\left\|T^{1}(t)-T^{0}(t)\right\| \frac{(b-a)^{i+n-1}}{(i+n-1) !} .
\end{aligned}
$$

This is a contraction operator whenever $\alpha<1$. By the Banach fixed point theorem $T$ has a unique fixed point and this fixed point is the unique solution of (1.1) and (1.2). To simplify our exposition, we omit the straightforward generalization to multipoint boundary value problems.

\section{CONCLUSIONS}

In this section, we obtain the general solution of

$$
T^{(n)}(t)=\sum_{r=0}^{n}\left(\begin{array}{l}
n \\
r
\end{array}\right) A^{n-r} T B^{r}
$$

in terms of the fundamental matrix solutions of $T^{\prime}=A T$ or $T^{\prime}=B^{*} T$. 
THEOREM 4.1 If $Y$ is a fundamental matrix solution of $T^{\prime}=A T$. Then $Y(n t)$ is a fundamental matrix solution of $T^{\prime}=(n A) T$.

THEOREM 4.2 If $Y$ is a fundamental matrix solution of $T^{\prime}=A T$ and $Z$ is a fundamental matrix solution of $T^{\prime}=B^{*} T$, then $T$ defined by

$$
T(t)=Y(n t) \mathcal{C} Z^{*}(m t)
$$

is a solution of

$$
T^{\prime}=(n A) T+T(m B) .
$$

Proof

$$
\begin{aligned}
T^{\prime}(t) & =n Y^{\prime}(n t) \mathcal{C} Z^{*}(n t)+m Y(n t) \mathcal{C} Z^{* \prime}(m t) \\
& =n A Y(n t) \mathcal{C} Z^{*}(n t)+m Y(m t) \mathcal{C} Z^{*} B \\
& =(n A) T+T(m B) .
\end{aligned}
$$

THEOREM 4.3 If $Y$ is a fundamental matrix solution of $T^{\prime}=A T$ and $Z$ is a fundamental matrix solution of $T^{\prime}=B^{*} T$ then $T$ defined by $T(t)=Y(n t) \mathcal{C} Z^{*}(n t)$ is a solution of

$$
T^{\prime}=(n A) T+T(n B)
$$

THEOREM 4.4 If $Y(t)$ is a fundamental matrix solution of $T^{\prime}=A T$ and $Z(t)$ is a fundamental matrix solution of $T^{\prime}=B^{*} T$ then $T(t)=$ $Y(n t) \mathcal{C} Z^{*}(n t)$ is a solution of the system

$$
\begin{aligned}
T^{(n)}(t)= & \left(n^{n} A\right) T+\left(\begin{array}{c}
n \\
1
\end{array}\right)\left(n^{n-1} A\right) T(n B)+\left(\begin{array}{l}
n \\
2
\end{array}\right) n^{n-2} A T\left(n^{2} B\right) \\
& +\cdots+\left(\begin{array}{c}
n \\
r
\end{array}\right)\left(n^{n-r} A\right) T\left(n^{r} B\right)+\cdots+T\left(n^{n} B\right) .
\end{aligned}
$$

We observe that $T$ defined by

$$
T(t)=Y(-2 t)\left(\mathcal{C}_{0}+\mathcal{C}_{1} t\right) Z^{*}(-2 t)
$$

is a solution of the matrix system

$$
T^{\prime \prime}(t)+4\left(A T^{\prime}+T^{\prime} B\right)+4\left(A^{2}+2 A T B+T B^{2}\right)=0 .
$$

Thus Theorem 1 given in [2] is a particular case of Theorem 4.4. 


\section{References}

[1] F.V. Atkinson, Discrete and Continuous Boundary Value Problems, Academic Press, New York (1964).

[2] S. Barnett, Matrix Differential Equations and Kronecker Products, SIAM J. Appl. Anal. 24(1) (1973).

[3] R. Bellman, Stability Theory of Differential Equations, McGraw-Hill, New York (1953).

[4] G.H. Golub, S. Nash and C.F. Van Loan, A Hessenberg-Schur method for the matrix problem $A X+X B=C$, IEEE Trans. Auto. Cont. AC-24: 909-913 (1979).

[5] K.W. Harald, Explicit solutions of the matrix equation $\sum_{i} A^{i} X D_{i}=\mathcal{C}^{*}, S I A M J$. Matrix Anal. Applic., 13(4): 1123-1130 (1992).

[6] K.N. Murty, G. Howell and S. Sivasundaram, Two (multi) point non-linear Lyapunov systems - existence and uniqueness, J. Math. Anal. Appl., 167, 505-515 (1992).

[7] E.L. Wachspress, The ADI Model Problem, Windsor, California (1995). 


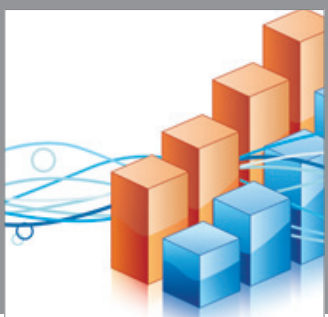

Advances in

Operations Research

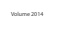

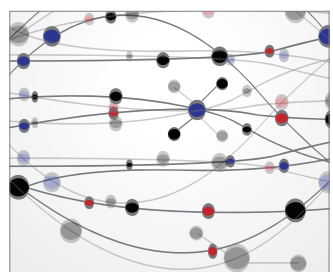

\section{The Scientific} World Journal
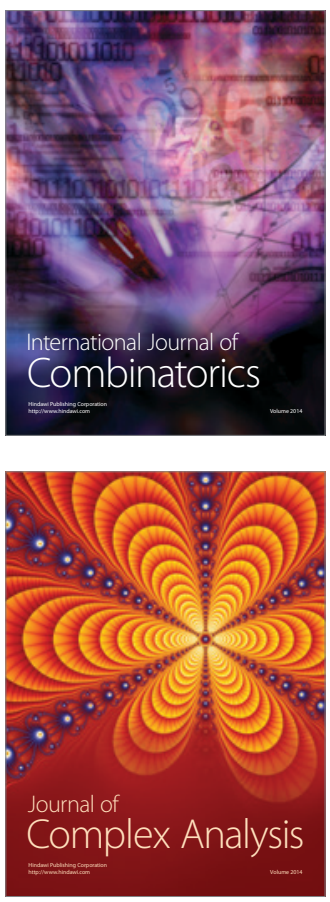

International Journal of

Mathematics and

Mathematical

Sciences
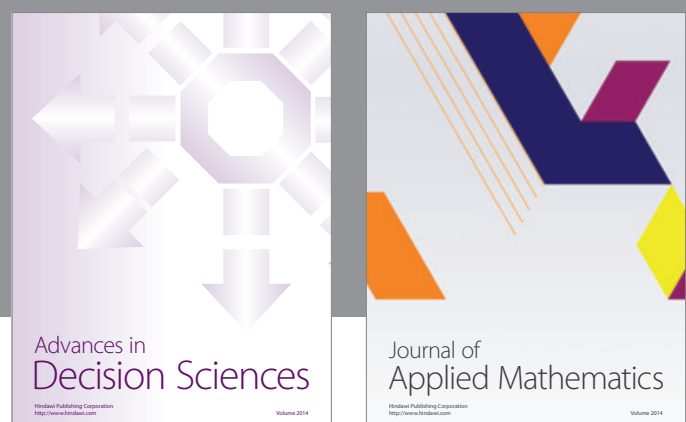

Journal of

Applied Mathematics
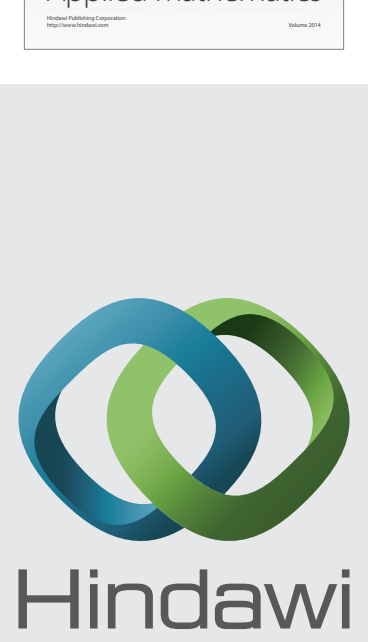

Submit your manuscripts at http://www.hindawi.com
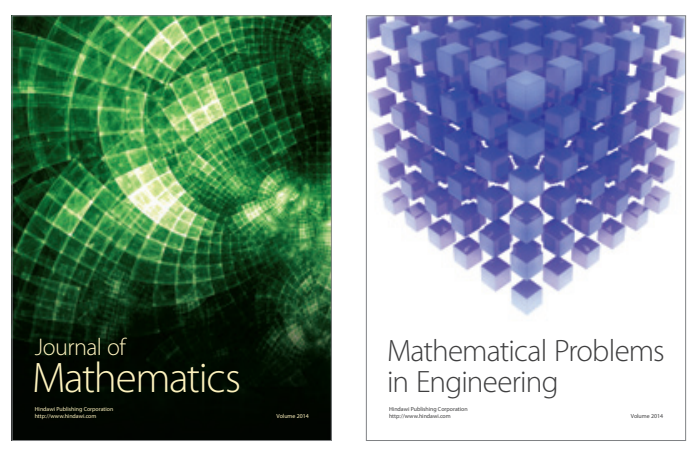

Mathematical Problems in Engineering
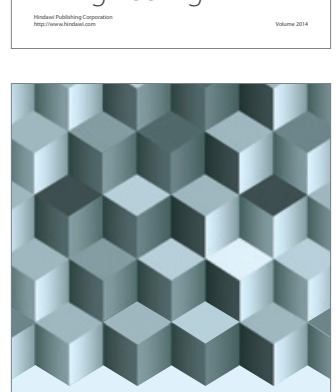

Journal of

Function Spaces
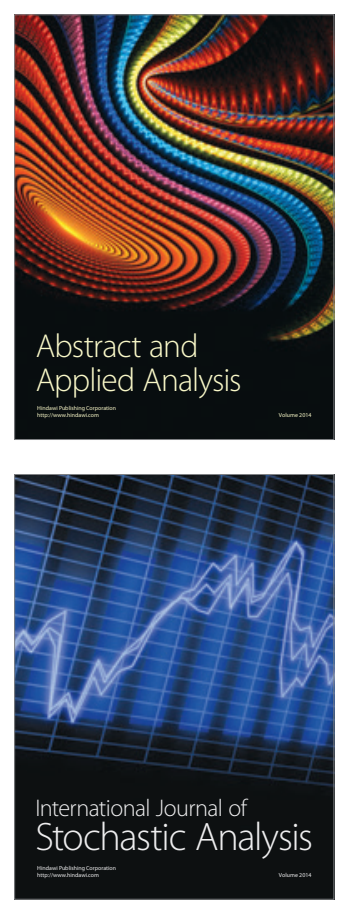

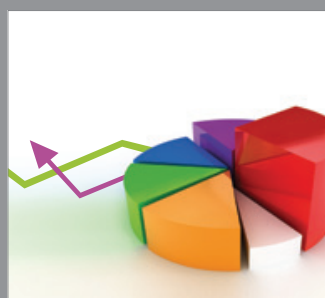

ournal of

Probability and Statistics

Promensencen
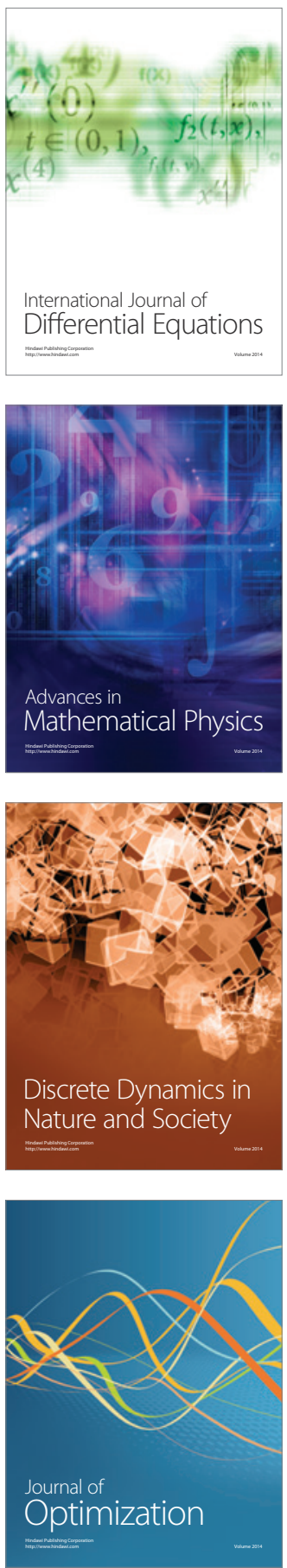